\title{
GRB 021004: Tomography of a gamma-ray burst progenitor and its host galaxy ${ }^{\star}$
}

\author{
A. J. Castro-Tirado ${ }^{1}$, P. Møller ${ }^{2}$, G. García-Segura ${ }^{3}$, J. Gorosabel ${ }^{1}$, E. Pérez ${ }^{1}$, A. de Ugarte Postigo ${ }^{4}$, E. Solano ${ }^{5}$,
} D. Barrado ${ }^{17,5}$, S. Klose ${ }^{6}$, D. A. Kann ${ }^{6}$, J. M. Castro Cerón ${ }^{7}$, C. Kouveliotou ${ }^{8}$, J. P. U. Fynbo ${ }^{9}$, J. Hjorth ${ }^{9}$, H. Pedersen ${ }^{9}$, E. Pian ${ }^{2} 10,11$, E. Rol ${ }^{12,13}$, E. Palazzi ${ }^{14}$, N. Masetti ${ }^{14}$, N. R. Tanvir ${ }^{12}$, P. M. Vreeswijk ${ }^{9}$, M. I. Andersen ${ }^{9}$, A. S. Fruchter ${ }^{15}$, J. Greiner ${ }^{16}$, R. A. M. J. Wijers ${ }^{13}$, and E. P. J. van den Heuvel ${ }^{13}$

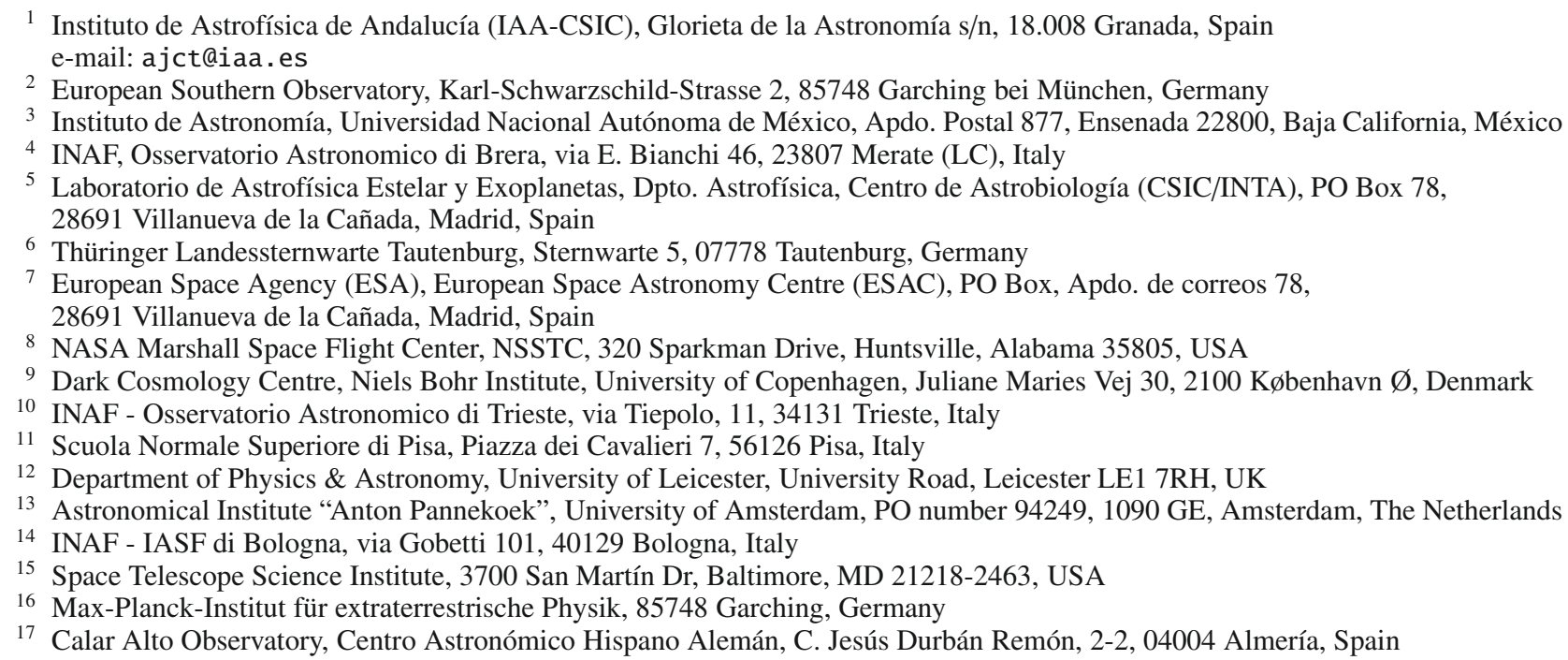

Received 24 December 2009 / Accepted 26 March 2010

ABSTRACT

\begin{abstract}
Aims. We analyse the distribution of matter around the progenitor star of gamma-ray burst GRB 021004 and the properties of its host galaxy with high-resolution echelle and near-infrared spectroscopy.

Methods. Observations were taken by the $8.2 \mathrm{~m}$ Very Large Telescope with the Ultraviolet and Visual Echelle spectrograph (UVES) and the Infrared Spectrometer And Array Camera (ISAAC) between 10 and $14 \mathrm{~h}$ after the onset of the event.

Results. We report the first detection of emission lines from a GRB host galaxy in the near-infrared, detecting $\mathrm{H} \alpha$ and the [O III] doublet. These allow us to independently measure the systemic redshift $(z=2.3304 \pm 0.0005)$, which is not contaminated by absorption as the $\operatorname{Ly} \alpha$ line is, and infer the host galaxy properties. From the visual echelle spectroscopy, we find several absorptionline groups spanning a range of about $3000 \mathrm{~km} \mathrm{~s}^{-1}$ in velocity relative to the redshift of the host galaxy. The absorption profiles are very complex with both velocity-broadened components extending over several $100 \mathrm{~km} \mathrm{~s}^{-1}$ and narrow lines with velocity widths of only $\sim 20 \mathrm{~km} \mathrm{~s}^{-1}$. By analogy with QSO absorption line studies, the relative velocities, widths, and degrees of ionization of the lines ("line-locking", "ionization-velocity correlation") show that the progenitor had both an extremely strong radiation field and several distinct mass-loss phases (winds).

Conclusions. These results are consistent with GRB progenitors being massive stars, such as luminous blue variables (LBVs) or Wolf-Rayet stars, providing a detailed picture of the spatial and velocity structure of the GRB progenitor star at the time of explosion. The host galaxy is a prolific star-forming galaxy with a $S F R$ of $\sim 40 M_{\odot} \mathrm{yr}^{-1}$.
\end{abstract}

Key words. gamma-ray bust: general - techniques: spectroscopic - stars: Wolf-Rayet - galaxies: starburst - cosmology: observations

\section{Introduction}

The afterglows of long-duration gamma-ray bursts (GRBs), which are linked to the explosions of massive stars (see Woosley \& Bloom 2006, for a review), are the most luminous optical sources in the universe for short periods of time (Kann et al. 2007; Bloom et al. 2009). Low-resolution optical spectroscopy

* Based on observations taken with the ESO's 8.2 m Very Large Telescope in Chile. only was initially usable for determining the redshift and placed them at cosmological distances (Metzger et al. 1997). Deeper insight came with the first medium-resolution spectrum, obtained with Keck ESI, of the afterglow of GRB 000926 (Castro et al. 2003). The first true high-resolution echelle spectra were obtained for GRB 020813 (Fiore et al. 2005), but they were of low signal-to-noise ratio (herafter $S / N$ ). The first echelle spectra of high $S / N$ were finally obtained for GRB 021004 , the focus of this work (see also Fiore et al. 2005). 
Spectroscopy allows deep insight into the environments of GRBs (Prochaska et al. 2006). Some highlights include the possible detection of a Galactic superwind in the host galaxy of GRB 030329 (Thöne et al. 2007), and variable absorption lines that result from direct UV pumping by the luminous GRB afterglow (Dessauges-Zavadsky et al. 2006; Vreeswijk et al. 2007; D'Elia et al. 2009). These detections have become possible for more afterglows due to the rapid localization capabilities of the Swift satellite (Gehrels et al. 2004) in combination with the rapid-response mode (RRM) now available for the Ultraviolet-Visual Echelle Spectrograph (UVES) at the Very Large Telescope (VLT) (e.g., Vreeswijk et al. 2007; D'Elia et al. 2009). The typical wavelength region has been increased to now extend from the ultraviolet into the $K$ band near-infrared (nIR) by the second-generation instrument X-Shooter at VLT (D'Odorico et al. 2006; see de Ugarte Postigo et al. 2010, for a first result).

GRB 021004 was detected at 12:06:14 universal time (UT) on 2002, October 4 with the gamma-ray instrument FREGATE, the wide-field X-ray Monitor (WXM) and the soft X-ray camera (SXC) aboard the High-Energy Transient Explorer (HETE-2) (Shirasaki et al. 2002). GRB 021004, was a moderately bright, long-duration $\left(T_{90}=100 \mathrm{~s}\right)$ event with fluences of $6.4 \times$ $10^{-7} \mathrm{erg} \mathrm{cm}^{-2}(7-30 \mathrm{keV})$ and $2.3 \times 10^{-6} \mathrm{erg} \mathrm{cm}^{-2}(30-400 \mathrm{keV})$ (Barraud et al. 2002).

The GRB was rapidly localized in-flight and the position was reported in less than a minute, allowing rapid groundbased follow-up that identified the fading optical afterglow of GRB 021004 (Fox et al. 2003). This prompted follow-up observations at many observatories, including extensive long-term coverage at X-rays (Sako \& Harrison 2002a,b), radio (Frail \& Berger 2002; Berger et al. 2002; Pooley 2002a,b) ${ }^{1}$, millimeter (de Ugarte Postigo et al. 2005), near-IR (de Ugarte Postigo et al. 2005; Fynbo et al. 2005), and optical wavelengths, both groundbased (Fox et al. 2003; Bersier et al. 2003; Uemura et al. 2003; Holland et al. 2003; Pandey et al. 2003; Mirabal et al. 2003; Kawabata et al. 2004; de Ugarte Postigo et al. 2005) and spacebased (Fynbo et al. 2005).

The isotropic energy release during the prompt emission of this GRB was modest, with $\log E_{\text {iso }}=52.65_{-0.17}^{+0.12}$ (Kann et al. 2010), and the dust extinction for this somehow reddish afterglow $(R-K \sim 3$ ) was also low as is typical of many wellobserved afterglows (Kann et al. 2006). Despite the low amount of energy that was promptly released, this is among the most luminous afterglows ever detected (Kann et al. 2006), even compared to a much larger Swift-era sample (Kann et al. 2010). The multiwavelength temporal evolution of the GRB 021004 afterglow can be explained by multiple energy injections (Björnsson et al. 2004; de Ugarte Postigo et al. 2005), although other scenarios cannot be discarded (Lazzati et al. 2002). GRB 021004 remains one of the most well-observed afterglows ever.

Early-time low and medium resolution spectroscopic observations allowed a redshift of $z=2.33$ to be determined on the basis of Ly- $\alpha$ absorption and emission lines (Chornock \& Filippenko 2002). Several absorption systems with outflow velocities of a few $1000 \mathrm{~km} \mathrm{~s}^{-1}$ were also reported (Salamanca et al. 2002; Savaglio et al. 2002) and studied in detail by Møller et al. (2002b), Wang et al. (2003), Matheson et al. (2003), Schaefer et al. (2003), Mirabal et al. (2003), Starling et al. (2005), and Lazzati et al. (2006). Jakobsson et al. (2005) also

\footnotetext{
1 See http://WwW.aoc.nrao.edu/ dfrail/grb021004. dat for the complete VLA data set.
}

report the detection of the host galaxy $\operatorname{Ly} \alpha$ line in narrow-band imaging.

Fiore et al. (2005) constrained the ionization parameters of the various absorption components detected above $\operatorname{Ly} \alpha$ (at an observer rest-frame of $4050 \AA$ ) in GRB 021004, interpreting them as density fluctuations, as Lazzati et al. (2002) did. Within the context of larger samples, the UVES spectra of this GRB were also studied by Chen et al. (2007) (the general lack of wind signatures in high-resolution spectra of GRB afterglows), Prochaska et al. (2008) (N V absorption lines toward GRB afterglows), Fox et al. (2008) (high-ionisation line systems toward GRB afterglows), Tejos et al. (2007, 2009), and Vergani et al. (2009) (study of Mg II foreground absorption systems).

The original expectation, as detailed high quality spectroscopy of GRB afterglows became possible, was that we would rapidly learn much about the GRB progenitors by studying the complex absorption line systems they were expected to exhibit due to ejection events leading up to the final collapse. This expectation has thus far not been met.

Despite the large number of long GRB afterglow spectra that have now been obtained, GRB 021004 continues to be one with the most complex set of intrinsic $\left(0-3000 \mathrm{~km} \mathrm{~s}^{-1}\right.$ ejection velocity) absorption systems. At the time, it also held the place as the lowest detected HI column density, and it still ranks between the lowest seven measured. Its complexity originally suggested that it represented a display of ejecta (Møller et al. 2002a; Mirabal et al. 2003; Fiore et al. 2005) but this interpretation was later disputed by Chen et al. (2007). A final interpretation of the complex systems has not yet been agreed on, and this object remains the most probable candidate to exhibit rarely detected signatures of events prior to the collapse. The rarity alone would warrant a more detailed discussion of the spectral features. In addition, we include UVES data below 4050 ang for a more detailed discussion of the absorption systems (Sect. 3.1) and nearIR VLT/ISAAC spectroscopy allowing a more accurate determination of host properties and redshift (Sect. 3.2). We discuss our results in the light of progenitor models for GRBs in Sect. 4, and summarize our work in Sect. 5.

For a Hubble constant of $H_{0}=72 \mathrm{~km} \mathrm{~s}^{-1} \mathrm{Mpc}^{-1}$, a matter density $\Omega_{\mathrm{m}}=0.3$, and a cosmological constant $\Omega_{\Lambda}=0.7$, the luminosity distance to the host is $d_{\mathrm{L}}=18.22 \mathrm{Gpc}$, and the lookback time is 10.42 Gyr. All errors are given at a $1 \sigma$ level of confidence for a parameter of interest unless stated otherwise.

\section{Observations and data reduction}

\subsection{Optical observations}

Observations were conducted with 8.2-m Very Large Telescope Units 1 (VLT/UT1, Antu) and 2 (VLT/UT2, Kueyen) at the European Southern Observatory (ESO) in Cerro Paranal (Chile). We obtained optical spectroscopy of the GRB afterglow starting 0.6 days after the burst using the Ultraviolet-Visual Echelle Spectrograph (UVES) on UT2. Contemporaneous nIR observations were taken using the Infrared Spectrometer And Array Camera (ISAAC) on UT1 (see Table 1 for a log of the observations).

The optical data reduction was performed using the UVES context running under MIDAS ${ }^{2}$. The UVES context is structured into reduction recipes allowing for data reduction in a semiautomatic manner. A master bias frame was used for the bias

2 MIDAS (Munich Imaging Data Analysis System) is developed and maintained by the European Southern Observatory (ESO). http:// www. eso.org/projects/esomidas/midas-distrib.html 
Table 1. Journal of the VLT GRB 021004 optical/nIR spectroscopic observations.

\begin{tabular}{clcccc}
\hline \hline $\begin{array}{c}\text { UT Time } \\
\text { (start) }\end{array}$ & Instr. & $\begin{array}{c}\text { Exp. } \\
\text { time (s) }\end{array}$ & $\begin{array}{c}\text { Spectral } \\
\text { range }(\AA)\end{array}$ & $\begin{array}{c}S / N \\
\text { ratio }^{a}\end{array}$ & $\begin{array}{c}\text { Resolution } \\
\lambda / \Delta(\lambda)\end{array}$ \\
\hline $03: 02$ & UVES & 3600 & $3290-4520$ & 1 & $\sim 56000$ \\
& & & $4620-5595$ & 2.2 & $\sim 51500$ \\
& & & $5675-6645$ & 2.7 & $\sim 51500$ \\
$03: 02$ & ISAAC & 3600 & $14200-18300$ & 5.2 & $\sim 500$ \\
$04: 10$ & UVES & 3600 & $3050-3870$ & 1 & $\sim 51300$ \\
& & & $4780-5755$ & 3.7 & $\sim 50500$ \\
& & & $5835-6805$ & 5.4 & $\sim 50500$ \\
$05: 15$ & UVES & \multirow{2}{*}{3600} & $3750-4650$ & 2.4 & $\sim 51500$ \\
& & & $6705-8520$ & 4.1 & $\sim 52300$ \\
$05: 27$ & ISAAC & 3000 & $8665-10400$ & 2.2 & $\sim 48700$ \\
\hline
\end{tabular}

Notes. All observations were obtained on October 5 (UT).

(a) Per resolution element.

Table 2. Individual high-velocity metal-lines systems in GRB 021004.

\begin{tabular}{|c|c|c|c|c|c|}
\hline Line ID & $\mathrm{C} 2$ & $\mathrm{C} 1$ & $\mathrm{D}$ & E2 & E1 \\
\hline Si III $\lambda 1,206$ & $\mathrm{y}^{a}$ & $\mathrm{y}$ & $\mathrm{y}$ & $\mathrm{y}$ & $\mathrm{y}$ \\
\hline H I Ly $\alpha \lambda 1,216$ & $\mathrm{y}$ & $\mathrm{y}$ & $\mathrm{y}$ & $\mathrm{y}$ & $\mathrm{y}$ \\
\hline N V $\lambda 1,238-1,242$ & $\mathrm{y}$ & - & - & - & $\mathrm{y}$ \\
\hline Si II $\lambda 1,260-1,264$ & - & $\mathrm{y}$ & $?$ & - & $\mathrm{y}$ \\
\hline C II $\lambda 1,334$ & $\mathrm{y}$ & $\mathrm{y}$ & - & - & - \\
\hline Si IV $\lambda 1,394-1,403$ & $?$ & $?$ & $?$ & $?$ & $\mathrm{y}$ \\
\hline C IV $\lambda 1,548-1,551$ & $\mathrm{y}$ & $\mathrm{y}$ & $\mathrm{y}$ & $\mathrm{y}$ & $\mathrm{y}$ \\
\hline $\mathrm{Al}$ II $\lambda 1,671$ & - & $\mathrm{y}$ & - & $\mathrm{y}$ & $\mathrm{y}$ \\
\hline Al III $\lambda 1,855-1,863$ & - & - & - & - & $\mathrm{y}$ \\
\hline Fe II $\lambda 2,344-2,382$ & - & - & - & - & $\mathrm{y}$ \\
\hline$z$ & 2.29671 & 2.29935 & 2.3216 & 2.3275 & 2.32891 \\
\hline$v\left(\mathrm{~km} \mathrm{~s}^{-1}\right)^{b}$ & $-3050^{c}$ & $-2810^{d}$ & $-795^{e}$ & $-262^{f}$ & $-134^{g}$ \\
\hline
\end{tabular}

Notes. ${ }^{(a)}$ y $=$ yes; $-=$ no; ? = doubtful, low significance due to low $S / N$ in the $\mathrm{S}$ IV region. ${ }^{(b)}$ Relative to the systemic velocity of the host galaxy at $z=2.3304$, which we set as zero. ${ }^{(c)}$ Embedded as $\mathrm{C} 1$ in the wide $\mathrm{C}$ complex (2730-3250 $\left.\mathrm{km} \mathrm{s}^{-1}\right)$. ${ }^{(d)}$ Two subcomponents at -2837 and $-2803 \mathrm{~km} \mathrm{~s}^{-1}$ (low ionization) and -2860 and $-2810 \mathrm{~km} \mathrm{~s}^{-1}$ (high ionization). ${ }^{(e)}$ Wide complex spanning $585-1005 \mathrm{~km} \mathrm{~s}^{-1} .{ }^{(f)}$ Embedded as E1 in the wide E complex (40-390 $\left.\mathrm{km} \mathrm{s}^{-1}\right) .{ }^{(g)}$ Two subcomponents at -150 and $-114 \mathrm{~km} \mathrm{~s}^{-1}$.

subtraction. The order tracing was performed taking advantage of a first guess calibration solution based on the UVES physical model. In all cases, except for the red spectrum centered on $8600 \AA$, which is affected by fringing effects, the flat field correction was applied after the order extraction. A Th-Ar lamp was used for wavelength calibration. The spectra were extracted using an optimal extraction method, which provides the object signal and the variance. After the extraction, the spectra were resampled to constant wavelength bins and the orders merged into a single spectrum. Finally, both the standard heliocentric and vacuum wavelength corrections were applied.

\subsection{Near-infrared observations}

For the nIR observations, we used the short wavelength mode in low resolution, which yields a spectral coverage of $1.42-1.83 \mu \mathrm{m}$ and $1.84-2.56 \mu \mathrm{m}$ in the $H$ and $K$ bands, respectively. The pixel scale of the ESO-Hawaii detector is 0.'146/pixel. For each band, 50 individual exposures of $60 \mathrm{~s}$ each were taken, shifting the target along the slit every 5 exposures in $H(3$ in $K$ ) to be able to remove the sky background, a standard technique in infrared astronomy. We also collected dark and flat field images, which were used in the reduction process performed with IRAF ${ }^{3}$. After combining the groups of exposures and extracting the individual spectra, we calibrated them using the air-glow $\mathrm{OH}$ emission lines present in the 2 dimensional images. This allows an accurate wavelength calibration with dispersions of 4 and $8 \AA /$ pix for $H$ and $K$, respectively. Finally, the individual spectra were mediancombined into a single one. In the case of the $H$ band spectrum, we used a G2 spectral type star to remove the telluric absorption bands. The spectra were flux-calibrated by means of the $J H K$ band observations that we obtained either side of each science spectral observation.

\section{Results}

\subsection{The blueshifted absorption-line systems}

The VLT/UVES data set (Fig. 1) exhibits a large number of absorption lines, as well as $\operatorname{Ly} \alpha$ in emission (see Table 3 for a list, and also Møller et al. 2002b; Starling et al. 2005; Jakobsson et al. 2005, on the Ly $\alpha$ emission line). Some of the absorption lines are due to two foreground systems at redshifts $z=1.3820$ and $z=1.6020$ (Møller et al. 2002b; Mirabal et al. 2003; see Tejos et al. 2007, 2009; and Vergani et al. 2009, for additional discussions about the foreground absorbers), but the remaining lines are from multiple systems found in the redshift range $2.2967 \leq z \leq 2.3291$.

In Fig. 2, we show the three wide absorption-line complexes in the redshift range $2.2967 \leq z \leq 2.3291$. The absorption profiles are plotted in velocity space and marked C, D, and E following the naming convention used by Møller et al. (2002a). The higher resolution of the UVES spectrum allowed us to identify four narrow line systems embedded inside the velocity structure of the broad systems $\mathrm{C}$ and $\mathrm{E}$. We name those systems $\mathrm{C} 1, \mathrm{C} 2$, $\mathrm{E} 1$, and E2. In the following, we discuss all of those systems in more detail in order of increasing velocity relative to the host redshift $(z=2.3304$, see Sect. 3.2).

E complex: The Ly $\alpha$ absorption width translates into velocities in the range $40-470 \mathrm{~km} \mathrm{~s}^{-1}$, while in C IV (where we observe the narrowest trough), the range is $40-390 \mathrm{~km} \mathrm{~s}^{-1}$. The difference in the high-velocity cut-offs (390 versus $470 \mathrm{~km} \mathrm{~s}^{-1}$ ) is most easily understood as a column density effect, i.e., the column density of the high velocity end is too low for C IV to be detectable. Alternatively, it could be caused by lower metallicity in the higher velocity gas of system E, or a different ionization. The simplest interpretation of the E complex is that it is caused by gas, spanning velocities from $40-470 \mathrm{~km} \mathrm{~s}^{-1}$ and densities decreasing from the slowest to the fastest moving part. Similar profiles have been observed in QSO broad absorption line (BAL) systems, where velocities up to $\approx 0.1 c$ have been found. BAL systems are interpreted as velocity broadening due to ejection and/or radiative acceleration. These BAL-like absorption troughs are not meaningfully fitted by standard (Voigt) profiles that assume a Gaussian velocity field.

Embedded in the E complex are two narrow-line systems at $134 \mathrm{~km} \mathrm{~s}^{-1}$ (E1) and $262 \mathrm{~km} \mathrm{~s}^{-1}$ (E2). The strongest component, $\mathrm{E} 1$, is divided equally into two subcomponents at $114 \mathrm{~km} \mathrm{~s}^{-1}$

${ }^{3}$ IRAF is the Image Reduction and Analysis Facility, a general purpose software system for the reduction and analysis of astronomical data. IRAF is written and supported by the IRAF programming group at the National Optical Astronomy Observatories (NOAO) in Tucson, Arizona, USA. 

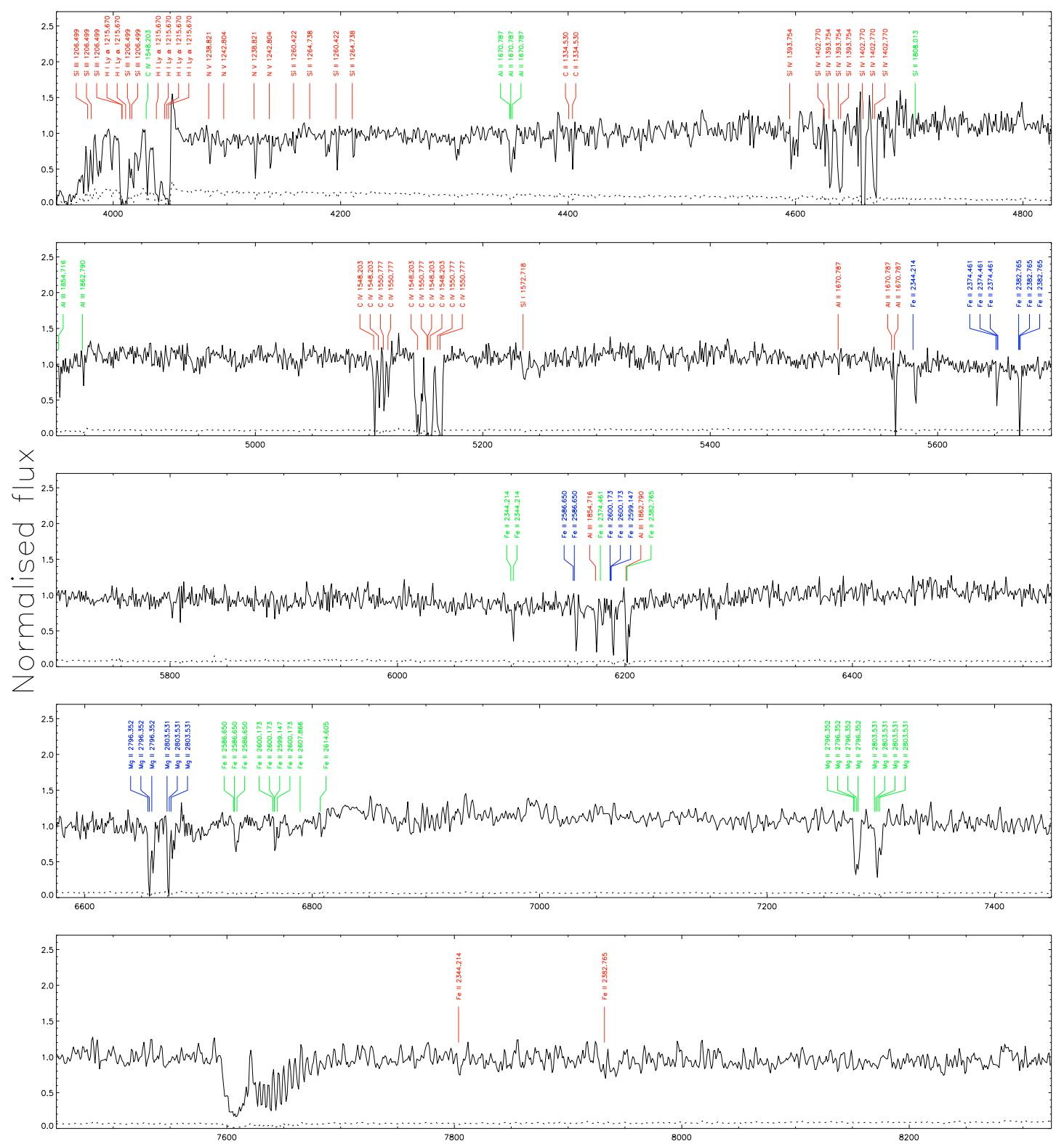

\section{Observed wavelength $(\AA)$}

Fig. 1. Overall view of GRB 021004 optical afterglow spectrum. These VLT/UVES data were obtained $\sim 0.6$ days after the GRB and show the Ly $\alpha$ emission line arising from the host galaxy redshift plus some of the most prominent absorption lines systems at (or very close to) the host galaxy redshift: low ionization lines (such as Fe II, Si II) and high ionization lines (such as Si IV, C IV, N V), all labelled in red colour. Also shown for completeness are the absorption systems for the foreground systems at $z=1.6020$ (labelled in green) and $z=1.3820$ (labelled in blue). For clarity, the original data have been smoothed. The telluric lines in the A-band (7600-7630 $\AA$ ) are noticeable. The dotted line is the error spectrum.

and $150 \mathrm{~km} \mathrm{~s}^{-1}$ (see Fig. 3) and exhibits absorption from a large number of both high and low ionic species (N V, C IV, Fe II, Al II, Al III, Si II, Si III, Si IV). The metal line absorption of E1 has similarities to so-called damped Ly $\alpha$ (DLA) systems, which in a few cases have been identified with high redshift, star-forming galaxies (Møller et al. 2002b) but it has too low HI column density along this sightline to be considered a DLA. Nevertheless, E1 is the system most likely to be identified with the interstellar medium (ISM) of the host galaxy of the GRB, whereby the velocity offset between the host and the ISM $\left(\sim 134 \mathrm{~km} \mathrm{~s}^{-1}\right)$ represents the velocity of the local absorbing cloud within the host. High and low ionic species are also seen in the second system (E2), but at lower significance. The two rightmost dotted vertical lines in Fig. 2 mark the systems E1 and E2.

D Complex: The D complex consists of a single, wide BALlike component seen in Ly $\alpha, \mathrm{C}$ IV, and Si III. Both the C IV $(1548 \AA)$ and the Ly $\alpha$ troughs provide a consistent relative velocity range from 585 to $1005 \mathrm{~km} \mathrm{~s}^{-1}$. We find residual flux at the bottom of both the Ly $\alpha$ and C IV (1548 $\AA$ ) troughs indicating that the gas is optically thin. We also see significant variations in the optical thickness as a function of relative velocity; in particular, one may note the two sharp "dips" marking shells of higher column density at the leading and the trailing edges. Those dips are seen in Ly $\alpha$, in both C IV lines and in Si III (marked by dotted lines in Fig. 2). The Si III trough is optically thick between those 
Table 3. Line Identifications in the GRB 021004 OA spectra.

\begin{tabular}{|c|c|c|c|}
\hline$\lambda_{\text {vacuum }}($ observed $)(\AA)$ & Line ID & $E W(\AA)^{a}$ & $z$ \\
\hline 4008 & H I Ly $\alpha 1215.670$ & & 2.2967 \\
\hline 4015 & Si III 1206.499 & & 2.3275 \\
\hline 4016 & Si III 1206.499 & & 2.3289 \\
\hline 4028 & C IV 1548.203 & & 1.6024 \\
\hline 4038 & H I Ly $\alpha 1215.670$ & & 2.3216 \\
\hline 4041 & H I Ly $\alpha 1215.670$ & & 2.3243 \\
\hline 4045 & H I Ly $\alpha 1215.670$ & & 2.3275 \\
\hline 4047 & H I Ly $\alpha 1215.670$ & & 2.3289 \\
\hline $4049^{b}$ & H I Ly $\alpha 1215.670$ & & 2.3304 \\
\hline 4084.0 & N V 1238.821 & $0.46 \pm 0.16$ & 2.2967 \\
\hline 4097.2 & N V 1242.804 & $0.35 \pm 0.15$ & 2.2967 \\
\hline 4123.9 & N V 1238.821 & $0.90 \pm 0.14$ & 2.3289 \\
\hline 4137.2 & N V 1242.804 & $0.66 \pm 0.12$ & 2.3289 \\
\hline 4158.5 & Si II 1260.422 & $0.25 \pm 0.10$ & 2.2993 \\
\hline 4195.8 & Si II 1260.422 & $0.71 \pm 0.19$ & 2.3289 \\
\hline 4210.2 & Si II 1264.738 & $0.51 \pm 0.16$ & 2.3289 \\
\hline 4347.4 & Al II 1670.787 & $0.49 \pm 0.17$ & 1.6020 \\
\hline 4399.6 & C II 1334.530 & $0.38 \pm 0.15$ & 2.2968 \\
\hline 4403.0 & C II 1334.530 & $0.79 \pm 0.14$ & 2.2994 \\
\hline 4639.7 & Si IV 1393.754 & $1.39 \pm 0.83$ & 2.3289 \\
\hline 4669.7 & Si IV 1402.770 & $1.29 \pm 0.46$ & 2.3289 \\
\hline 5104.0 & C IV 1548.203 & $1.77 \pm 0.12$ & 2.2967 \\
\hline 5107.8 & C IV 1548.203 & $0.16 \pm 0.03$ & 2.2992 \\
\hline 5108.5 & C IV 1548.203 & $0.84 \pm 0.10$ & 2.2998 \\
\hline 5112.5 & C IV 1550.777 & $1.15 \pm 0.09$ & 2.2967 \\
\hline 5116.2 & C IV 1550.777 & $0.06 \pm 0.04$ & 2.2992 \\
\hline 5117.0 & C IV 1550.777 & $0.67 \pm 0.08$ & 2.2998 \\
\hline 5138.3 & C IV 1548.203 & & 2.3189 \\
\hline 5140.0 & C IV 1548.203 & & 2.3200 \\
\hline 5143.1 & C IV 1548.203 & & 2.3220 \\
\hline 5146.7 & C IV 1550.777 & & 2.3188 \\
\hline 5146.7 & C IV 1548.203 & & 2.3243 \\
\hline 5148.5 & C IV 1550.777 & & 2.3200 \\
\hline 5148.5 & C IV 1548.203 & & 2.3255 \\
\hline 5151.7 & C IV 1550.777 & & 2.3220 \\
\hline 5152.9 & C IV 1548.203 & & $2.3275+2.3289$ \\
\hline 5155.2 & C IV 1550.777 & & 2.3243 \\
\hline 5157.6 & C IV 1550.777 & & 2.3255 \\
\hline 5161.5 & C IV 1550.777 & & $2.3275+2.3289$ \\
\hline 5235.4 & Si I 1572.718 & $0.80 \pm 0.10$ & 2.3289 \\
\hline 5559.5 & Al II 1670.787 & $0.23 \pm 0.07$ & 2.3275 \\
\hline 5561.9 & Al II 1670.787 & $1.73 \pm 0.12$ & 2.3289 \\
\hline 5578.0 & Fe II 2344.214 & $1.01 \pm 0.15$ & 1.3796 \\
\hline 5651.2 & Fe II 2374.461 & $0.52 \pm 0.06$ & 1.3800 \\
\hline 5652.4 & Fe II 2374.461 & $0.16 \pm 0.05$ & 1.3805 \\
\hline 5652.9 & Fe II 2374.461 & $0.13 \pm 0.02$ & 1.3807 \\
\hline 5671.2 & Fe II 2382.765 & $0.86 \pm 0.07$ & 1.3801 \\
\hline 5671.9 & Fe II 2382.765 & $0.41 \pm 0.06$ & 1.3804 \\
\hline 5672.6 & Fe II 2382.765 & $0.37 \pm 0.06$ & 1.3807 \\
\hline 6099.6 & Fe II 2344.214 & $0.59 \pm 0.04$ & 1.6020 \\
\hline 6101.7 & Fe II 2344.214 & $0.28 \pm 0.04$ & 1.6029 \\
\hline 6154.5 & Fe II 2586.650 & $0.67 \pm 0.08$ & 1.3794 \\
\hline \multirow[t]{2}{*}{6155.0} & Fe II 2586.650 & $0.50 \pm 0.09$ & 1.3798 \\
\hline & Fe II 2365.552 & $0.50 \pm 0.09$ & 1.6020 \\
\hline 6174.2 & Al III 1854.716 & $1.15 \pm 0.09$ & 2.3289 \\
\hline 6178.0 & Fe II 2374.461 & $0.52 \pm 0.08$ & 1.6020 \\
\hline 6187.0 & Fe II 2600.173 & $0.67 \pm 0.10$ & 1.3794 \\
\hline 6187.5 & Fe II 2600.173 & $0.44 \pm 0.08$ & 1.3798 \\
\hline 6188.0 & Fe II 2599.147 & $0.50 \pm 0.09$ & 1.3808 \\
\hline 6201.0 & Al III 1862.790 & $0.73 \pm 0.12$ & 2.3289 \\
\hline 6202.0 & Fe II 2382.765 & $0.94 \pm 0.16$ & 1.6028 \\
\hline 6654.0 & Mg II 2796.352 & $1.95 \pm 0.11^{*}$ & 1.3796 \\
\hline 6655.5 & Mg II 2796.352 & $1.95 \pm 0.11^{*}$ & 1.3802 \\
\hline 6657.0 & Mg II 2796.352 & $0.71 \pm 0.08$ & 1.3808 \\
\hline 6671.0 & Mg II 2803.531 & $1.89 \pm 0.41^{*}$ & 1.3796 \\
\hline 6673.0 & Mg II 2803.531 & $1.89 \pm 0.41 *$ & 1.3802 \\
\hline 6729.0 & Fe II 2586.650 & $0.25 \pm 0.07$ & 1.6016 \\
\hline 6730.2 & Fe II 2586.650 & $0.60 \pm 0.07$ & 1.6020 \\
\hline 6732.5 & Fe II 2586.650 & $0.18 \pm 0.06$ & 1.6029 \\
\hline 6764.0 & Fe II 2600.173 & $0.56 \pm 0.10$ & 1.6015 \\
\hline \multirow[t]{2}{*}{6765.5} & Fe II 2600.173 & $0.71 \pm 0.09 *$ & 1.6020 \\
\hline & Fe II 2599.147 & $0.71 \pm 0.09 *$ & 1.6030 \\
\hline 6768.0 & Fe II 2600.173 & $0.46 \pm 0.09$ & 1.6029 \\
\hline 6788.0 & Fe II 2607.866 & $0.14 \pm 0.05$ & 1.6030 \\
\hline
\end{tabular}

Table 3. continued.

\begin{tabular}{lccc}
\hline \hline$\lambda_{\text {vacuum (observed) }(\AA)}$ & Line ID & $E W(\AA)^{a}$ & $z$ \\
\hline 7274.2 & Mg II 2796.352 & $1.11 \pm 0.10$ & 1.6015 \\
7275.5 & Mg II 2796.352 & $0.83 \pm 0.08$ & 1.6020 \\
7277.0 & Mg II 2796.352 & $0.44 \pm 0.07$ & 1.6025 \\
7278.5 & Mg II 2796.352 & $0.83 \pm 0.08$ & 1.6029 \\
7293.0 & Mg II 2803.531 & $0.89 \pm 0.09$ & 1.6015 \\
7294.5 & Mg II 2803.531 & $0.98 \pm 0.09$ & 1.6020 \\
7296.0 & Mg II 2803.531 & $0.24 \pm 0.06$ & 1.6025 \\
7297.0 & Mg II 2803.531 & $1.00 \pm 0.10$ & 1.6029 \\
7803.7 & Fe II 2344.214 & $0.71 \pm 0.28$ & 2.3289 \\
7931.8 & Fe II 2382.765 & $0.46 \pm 0.21$ & 2.3288 \\
$16520^{c}$ & [O III] 4960.295 & $-(16 \pm 7)$ & 2.3304 \\
$16679^{d}$ & [O III] 5008.240 & $-(21 \pm 6)$ & 2.3304 \\
$21863^{e}$ & H I 6564.610 & $-(37 \pm 10)$ & 2.3304 \\
\hline
\end{tabular}

Notes. ${ }^{(a)}$ Gaussian profile fitting (for non saturated lines only). Star symbols refer to complex systems. ${ }^{(b)}$ Emission line. Partially absorbed, Ly $\alpha$ flux $=(1.5 \pm 0.2) \times 10^{-16} \mathrm{erg} \mathrm{cm}^{-2} \mathrm{~s}^{-1}{ }^{\left({ }^{c}\right)}$ Emission line [O III] flux $=(4 \pm 3) \times 10^{-17} \mathrm{erg} \mathrm{cm}^{-2} \mathrm{~s}^{-1}$. (d) Emission line [O III] flux = $(12 \pm 3) \times 10^{-17} \mathrm{erg} \mathrm{cm}^{-2} \mathrm{~s}^{-1}$. ${ }^{(e)}$ Emission line $\mathrm{H} \alpha$ flux $=(11 \pm 2) \times$ $10^{-17} \mathrm{erg} \mathrm{cm}^{-2} \mathrm{~s}^{-1}$.

two edges, but then appears to have an optically thin extension towards lower ejection velocities. However, this trough overlaps with $\operatorname{Ly} \alpha$ of system $\mathrm{C}$, and the extension is found to belong to system C (see below).

It is remarkable that the $\mathrm{D}$ and $\mathrm{E}$ systems are separated in velocity by exactly the amount required to shift the C IV $1550 \AA$ line complex right on top of the C IV $1548 \AA$ one in the spectrum (Møller et al. 2002b; Savaglio et al. 2002). This shift is known as absorption-absorption "line-locking" and is not uncommon in QSO spectra where radiative acceleration is important (e.g., Srianand et al. 2002). The line-locking between the D and E systems strongly suggests that a process similar to that seen in intrinsic QSO absorption systems is indeed involved here, i.e., radiative acceleration.

$C$ Complex: As discussed above, the wide Ly $\alpha$ trough of system $\mathrm{C}$ is partly caused by $\mathrm{Si}$ III absorption from system D, but also contains Ly $\alpha$ absorption of a wide component spanning the velocity range $2730-3250 \mathrm{~km} \mathrm{~s}^{-1}$, because we detect C II, C IV, Si II, Si III, Al II, and N V absorption in this velocity range (Fig. 2). The finely tuned velocity offset causing a precise overlap between the Ly $\alpha$ (C complex) and Si III (D complex) lines could be due to chance coincidence (although with small probability, see below). Given the evidence of line-locking between the two C IV lines between systems D and E, the overlap is most likely the result of radiative acceleration leading to linelocking of two expanding shells corresponding to the $\mathrm{C}$ and $\mathrm{D}$ complexes.

Embedded in $\mathrm{C}$, we identify two narrow systems at $3050 \mathrm{~km} \mathrm{~s}^{-1}$ (C2) and at $\sim 2810 \mathrm{~km} \mathrm{~s}^{-1}$ (C1). C2 is a high ionisation system that exhibits strong $\mathrm{N} \mathrm{V}$ and $\mathrm{C}$ IV absorption, but no absorption from singly ionized ions. $\mathrm{C} 1$ is a low ionization system with moderate C IV absorption and clear detections of the singly ionized species Al II and Si II, which usually identifies a cloud optically thick at the Lyman limit (see Table 3). It is most likely that $\mathrm{C} 2$, although farthest away from the host redshift in velocity space, corresponds to the part of the wind that is nearest to the progenitor site in physical space. This ionizationvelocity correlation is also observed in QSOs: the high ionization lines are often seen at the highest relative velocities for the $z_{\mathrm{abs}} \approx z_{\mathrm{em}}$ absorption systems in QSO spectra (e.g., Møller et al. 1994). As for E1, C1 consists of two subcomponents, detected at $2803 \mathrm{~km} \mathrm{~s}^{-1}$ and $2837 \mathrm{~km} \mathrm{~s}^{-1}$ (low ionization) and at 


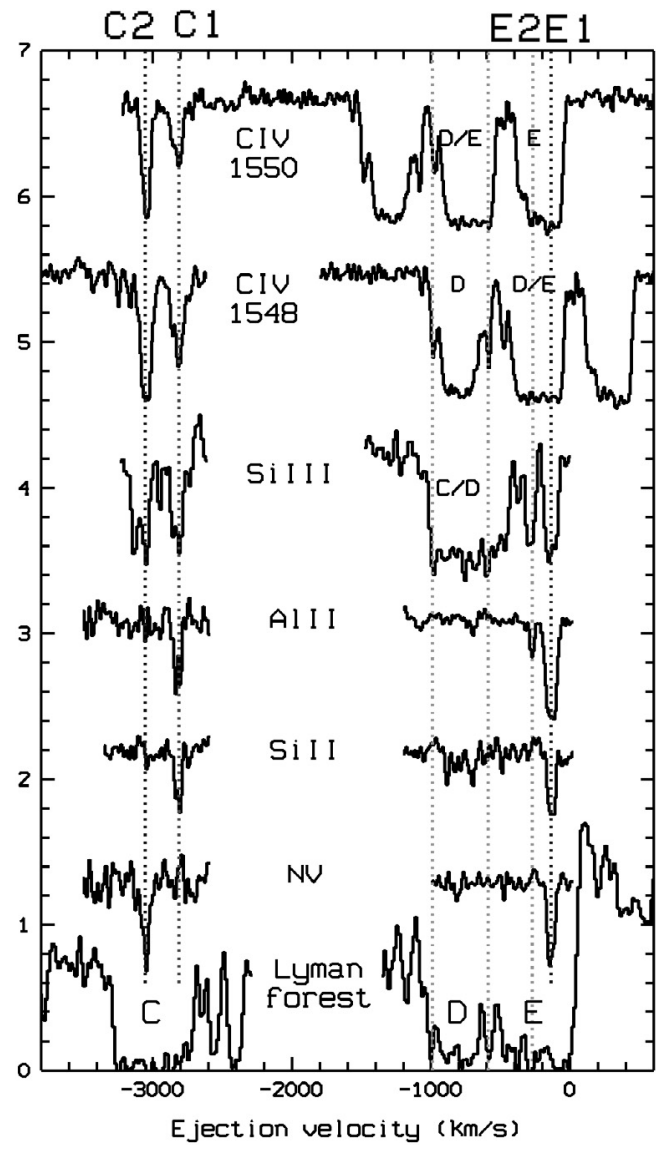

Fig. 2. In this figure, we have converted the observed wavelengths into velocities relative to the host redshift $(z=2.3304)$ for each ionic species significantly detected. Ly $\alpha$ is plotted at the bottom. To optimize the signal-to-noise ratio, two lines of N V (1238 and $1242 \AA)$ and four lines of Si II $(1260,1264,1533$, and $1816 \AA)$ have been coadded. The remaining lines are all single. The absorption components around $z \sim 2.3$ observed at velocities up to $3250 \mathrm{~km} \mathrm{~s}^{-1}$ are indicative of fast winds, ejected by a hot and massive stellar progenitor. The D and E complexes both contain velocity-broadened, BAL-like components. Within the E complex, there are narrow lines, E1 and E2, presumably from the ISM of the host galaxy. There are also narrow shells in the high-velocity system $\mathrm{C}, \mathrm{C} 1$, and $\mathrm{C} 2$. The dotted lines indicate the positions of E1, E2, the "dips" at the leading and the trailing edges of D, and the $\mathrm{C} 1$ and $\mathrm{C} 2$ systems.

$2810 \mathrm{~km} \mathrm{~s}^{-1}$ and $2860 \mathrm{~km} \mathrm{~s}^{-1}$ (high ionization). We again observe the ionization-velocity correlation seen already between C1 and C2 (Fig. 2). In a similar way to Fiore et al. (2005), we do consider $\mathrm{C} 1$ as part of the ejected systems simply on the basis of the low probability that is extrinsic, although we cannot exclude its association with the neighbouring galaxy detected by HST (Chen et al. 2007). In that case, it should be realized that the comparatively low H I column density reported by Fynbo et al. (2005) would be the sum of the H I column of the two galaxies, such that they both have even lower H I columns.

\subsection{The near-IR emission lines}

Assuming a redshift of the host of $z=2.3351$ (Møller et al. 2002 b, based on Ly $\alpha$ emission), we searched the ISAAC spectra for redshifted lines of $\mathrm{H} \mathrm{I}(\mathrm{H} \alpha, \mathrm{H} \beta)$ and [O III] (4959 $\mathrm{\AA} / 5007 \AA)$. There are tentative detections at low significance of $\mathrm{H} \alpha$ and the [O III] lines. To confirm the reality of the lines, we used the method that we introduced in Jensen et al. (2001). Our method
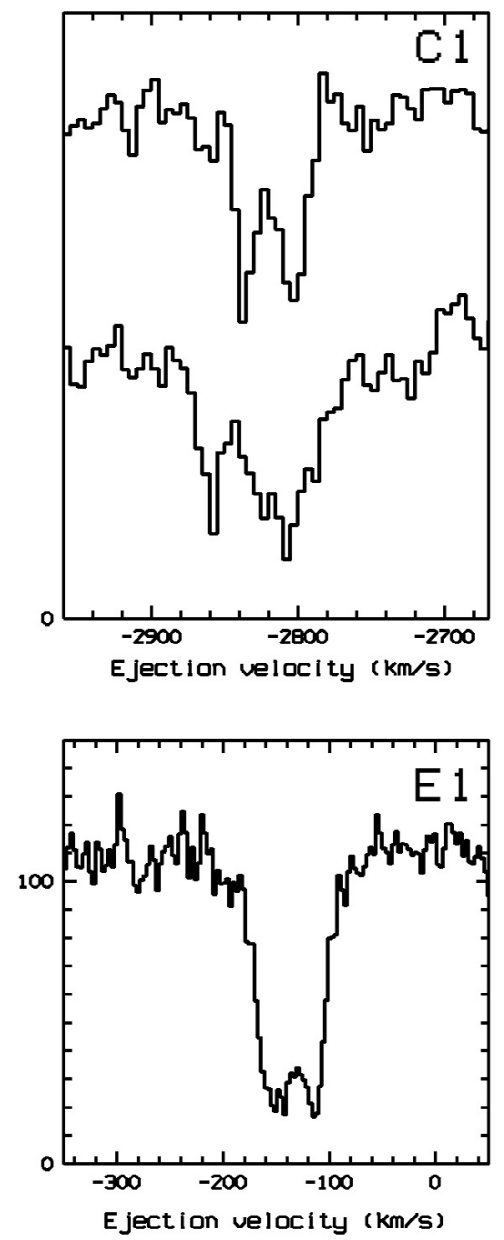

Fig. 3. A zoomed image of the high-resolution VLT/UVES spectrum at the location of the $\mathrm{C} 1$ and $\mathrm{E} 1$ narrow-line absorption systems. The data represent the coadded spectra of the different ions $(\mathrm{N} V+\mathrm{Si} I I+$ $\mathrm{Al}$ II $+\mathrm{Al}$ III) in velocity space. Thus, $\mathrm{C} 1$ has two subcomponents at $2803 \mathrm{~km} \mathrm{~s}^{-1}$ and $2837 \mathrm{~km} \mathrm{~s}^{-1}$ (low ionization, Si II + Al II, top) and $2810 \mathrm{~km} \mathrm{~s}^{-1}$ and $2860 \mathrm{~km} \mathrm{~s}^{-1}$ (high ionization, Si III + C IV, bottom). The ionization-velocity correlation also seen in QSOs (Møller et al. 1994) and WR nebulae (Smith et al. 1984) is noticeable. E1 divides equally into two subsystems at $114 \mathrm{~km} \mathrm{~s}^{-1}$ and $150 \mathrm{~km} \mathrm{~s}^{-1}$.

briefly involves first rebinning the spectra into redshifts for the rest wavelength of each expected line, in our case the lines of $\mathrm{H} \alpha$ and [O III] (4959 $\AA / 5007 \AA$ ). The three "redshift spectra" were then coadded (with weights 2,1 , and 1 because of the slightly higher $S / N$ of the tentative $\mathrm{H} \alpha$ detection). The resulting combined line is clearly detected, as shown in Fig. 4.

To this combined line, we fitted a Gaussian (overplotted as a blue dotted line) and measured the systemic redshift to be $z=$ $2.3304 \pm 0.0005$, which is significantly different from the values derived from the Ly $\alpha$ emission line (which may be absorbed or modified by resonant scattering) or from the absorption systems (which are affected by the local velocity of the absorbing cloud in the host galaxy). The width of the Gaussian was found to be exactly the spectral resolution, so the lines are unresolved and we can place an upper limit on the intrinsic line width of $600 \mathrm{~km} \mathrm{~s}^{-1}$ FWHM.

Using a redshift of 2.3304 and the width of the resolution profile, we then fitted Gaussian profiles to the data (the continuum level and the amplitude of the Gaussian being the only free parameters) using min-square deviation. For the two [O III] lines, we also imposed a 1:3 flux ratio (Osterbrock 1989), we found 

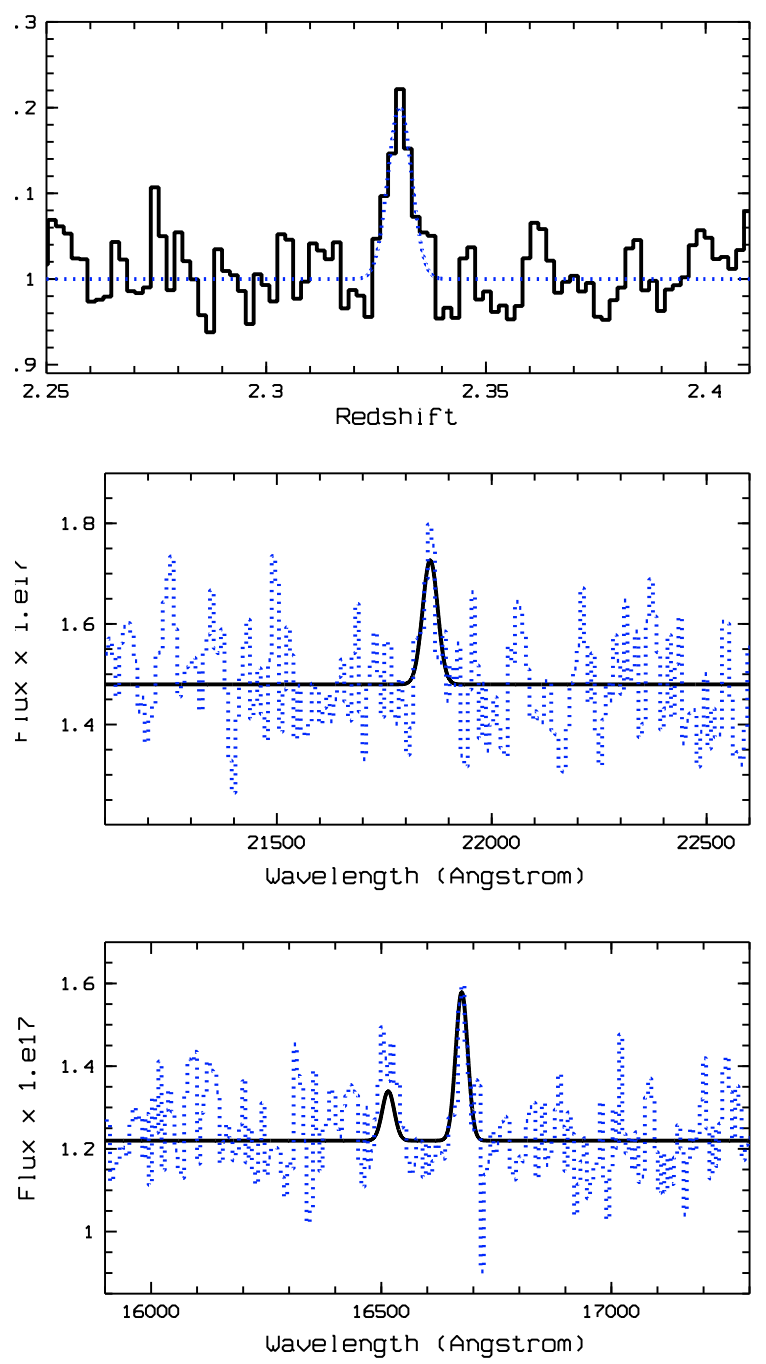

Fig. 4. Bottom panels: a zoomed image of the GRB 021004 nIR afterglow spectrum. These VLT/ISAAC data were obtained $\sim 0.6$ days after the GRB and show the $\mathrm{H} \alpha$ and [O III] emission lines arising from the host galaxy. Top panel: the coaddition of the three redshift spectra (as explained in the text) is shown, providing an independent measurement of the systemic redshift $z=2.3304$ based on the nIR line emission. In the middle and bottom panels, we show the optimal fits (black), and the data itself (blue dotted).

the following fluxes: flux $=(4 \pm 1) \times 10^{-17} \mathrm{erg} \mathrm{cm}^{-2} \mathrm{~s}^{-1}([\mathrm{O}$ III] $4959 \AA),(12 \pm 3) \times 10^{-17} \mathrm{erg} \mathrm{cm}^{-2} \mathrm{~s}^{-1}([\mathrm{O} \mathrm{III}] 5007 \AA)$, and $(11 \pm 2) \times 10^{-17} \mathrm{erg} \mathrm{cm}^{-2} \mathrm{~s}^{-1}(\mathrm{H} \alpha)$. The $\mathrm{H} \beta$ flux is consistent with zero.

\section{Discussion}

Following the suggestion that there is often a link between long-duration GRBs and core-collapse supernovae (Stanek et al. 2003; Hjorth et al. 2003) as proposed by Woosley (1993) we know that GRBs represent the end stages of massive-star evolution. As we now describe, the results presented here provide independent evidence of a massive star $\left(\geq 40 M_{\odot}\right)$ progenitor and additional insight into the immediate surroundings and prior evolution of the star leading up to the catastrophic event.

\subsection{A LBV / Wolf-Rayet progenitor}

The probability that the three absorption systems $\mathrm{C} 1, \mathrm{C} 2$, and D lie in front of the GRB host galaxy as a chance coincidence is very small and can be computed using the number density of intervening $\mathrm{C}$ IV absorbers at $1.8<z<3.5$ of $1.5 \pm 0.2$ per unit redshift (Sargent et al. 1998). We find that the probability of finding three of them within the redshift range $\Delta z=0.0337$ spanned by C1, C2, and D is $\sim 10^{-4}$. Fiore et al. (2005) used a similar argument but added the column densities to obtain an even lower probablility. Like Fiore et al. (2005), we conclude that the absorbers must be intrinsic to the host galaxy. The large blueshift then makes it unlikely that the physical location can be anywhere else than close to the GRB progenitor.

Furthermore, the probability that the C IV $1550 \AA$ line for complex E falls on top of the C IV $1548 \AA$ line for complex D and that the $\operatorname{Ly} \alpha$ line for system $\mathrm{C}$ falls on top of the Si III line for system D by chance is small, of order of a few percent for each, although difficult to quantify due to the complex profiles. Therefore, it makes sense to seek a physical explanation of the complex structure of the $\mathrm{C}, \mathrm{D}$, and $\mathrm{E}$ systems.

The multiple absorption-line systems found in the spectrum of GRB 021004 are naturally explained by multiple shell structures formed by the stellar winds of a massive progenitor star (Schaefer et al. 2003). These line-driven winds are expected for very massive stars (Castor et al. 1975; García Segura et al. 1996) that end their lives as Wolf-Rayet stars (WR) (de Koter et al. 1997), after passing through an unstable luminous blue variable (LBV) phase (Langer et al. 1999). Stellar evolution calculations for massive stars predict a number of different phases in which the wind properties associated with those phases, i.e., the velocities and mass-loss rates, become strongly time-varying (Langer et al. 1999; Maeder 1983). The minimum initial mass for a star to become a LBV is $\sim 40 M_{\odot}$ (Langer et al. 1994; Maeder \& Meynet 2000). During the course of their evolution, starting as O-class stars $(\mathrm{O} \rightarrow \mathrm{LBV} \rightarrow \mathrm{WR} \rightarrow \mathrm{SN})$, stars have distinct winds in which their velocities are proportional to the escape velocities. Thus, for each transition where a fast wind (i.e., when the stellar radius was small) follows a slow one (larger stellar radius), a shell of swept-up material is formed. Within this simple scheme, computations of the circumstellar medium around a $60 M_{\odot}$ star at the zero-age main sequence (García Segura et al. 1996) may produce the number of distinct compressed shells in the kinematic range seen in Fig. 2.

During the LBV phase, when hydrogen shell burning occurs, the stellar models are affected by several instabilities that cannot yet be modelled self-consistently and thus the unstable LBV phase cannot be fully resolved (Langer et al. 1994). However, several LBV observations show that a number of different swept-up shells can be formed. In particular, the D complex discussed above is akin to the Homunculus nebula around $\eta$ Car, which contains $\sim 1 M_{\odot}$ and expands at a speed in the range $700-1000 \mathrm{~km} \mathrm{~s}^{-1}$ (Humphreys \& Davidson 1994). As the formation of each of these swept-up shells occurs when the LBV reaches the Eddington limit (Langer et al. 1999), massive stars are able to form several compressed shells before ending their lives.

We note that, since the LBV stellar wind is asymmetric and often leads to a bipolar or at least asymmetric nebula (Gruendl et al. 2000), the GRB has to be beamed in the same direction as the wind for us to see the shells in absorption. Similar, but less spectacular, complex absorption has been detected in GRB 030226 (Klose et al. 2004; but see Shin et al. 2006) and GRB 050505 (Berger et al. 2006); this effect is not seen, however, in several other cases with similar quality data (Chen et al. 2007). It is possible, therefore, that GRBs for which no such complex velocity structure is seen have different relative orientations of 


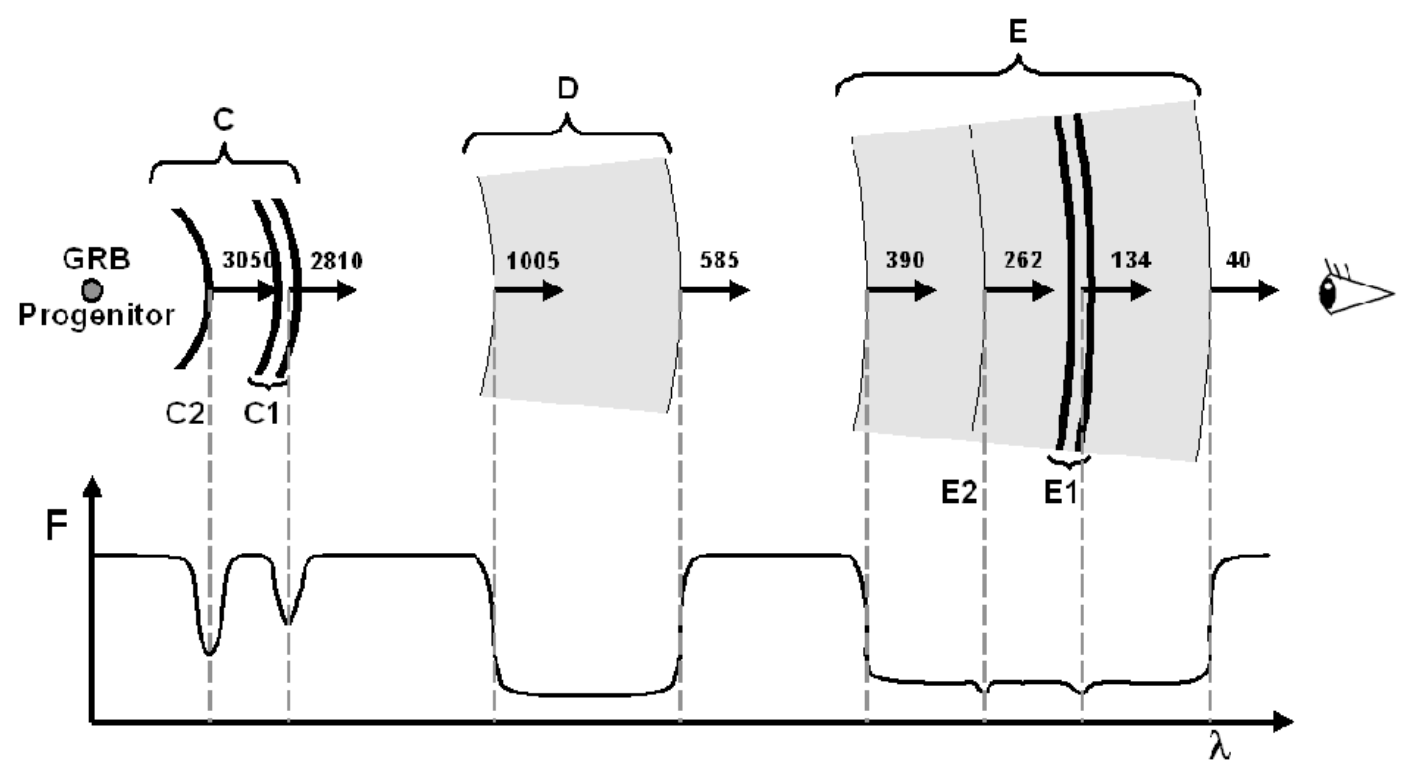

Fig. 5. A sketch showing the absorption-line complexes C, D, and E along the line of sight to GRB 021004, not drawn to scale. Velocities are given in $\mathrm{km} \mathrm{s}^{-1}$. Complex $\mathrm{C}$, with the highest relative velocity, is most likely the closest to the progenitor site in space and is probably formed during the WR phase. The high-ionization sub-component $\mathrm{C} 2$ is the closest one to the progenitor whereas the colder sub-component $\mathrm{C} 1$ is further out and we cannot exclude that it could even be related to the neighbouring galaxy detected by HST (Chen et al. 2007). Complex E has the lowest relative velocity, and the broad part of the $\mathrm{E}$ complex is most likely caused by the oldest wind formed when the progenitor evolved from being an $\mathrm{O}$ star into the LBV phase or in the early LBV phase. The narrow E1 component has the properties expected for the ISM of the host galaxy. We believe that the D complex is caused by winds formed during the unstable LBV phase.

the wind and GRB jet. Highly ionized gas $\left(\sim T=10^{5} \mathrm{~K}\right)$ of e.g., C IV, N V or Si IV, similar to that found in the environment of GRB 021004, has already been reported in a number of galactic ring nebulae surrounding Wolf-Rayet stars (Boronson et al. 1997). To form these gas shells, forward shocks moving at $\sim 10^{2} \mathrm{~km} \mathrm{~s}^{-1}$, or reverse shocks at $\sim 10^{2}-10^{3} \mathrm{~km} \mathrm{~s}^{-1}$ are required. The most natural explanation of the $\mathrm{C}$ complex is that it was formed during the WR phase, with $\mathrm{C} 1$ and $\mathrm{C} 2$ lying in an expanding free wind, before reaching the shocked stellar wind. The ionization-velocity correlation seen in the systems $\mathrm{C} 1$ and C2 has also been detected in WR nebulae, but at lower velocities ( 100-150 $\mathrm{km} \mathrm{s}^{-1}$; Smith et al. 1984).

In addition to the detailed picture of the spatial and velocity structure of the GRB progenitor star at the time of explosion (Fig. 5), our observations have also provided insight into the physical mechanism producing the progenitor shell structure. The $\mathrm{C} 1$ and $\mathrm{C} 2$ profiles are not P-Cygni, but almost symmetric profiles, thus ruling out their being very close to the burst (within $\sim 0.2 \mathrm{pc}$ ) and accelerated by the GRB ionizing flux to the observed velocities, as has been proposed elsewhere (Schaefer et al. 2003; Mirabal et al. 2003). The detected line-locking also favours radiative acceleration by the progenitor star.

\subsection{A starburst host galaxy}

An extremely blue host galaxy was detected in late-time imaging (Fynbo et al. 2005; de Ugarte Postigo et al. 2005; Jakobsson et al. 2005). To derive the star-formation rate (SFR), we studied the emission lines seen in the combined optical-nIR spectrum.

The emission line parameters were measured by applying a Gaussian fit to the emission line and a flat fit to the continuum. If all the $\mathrm{H} \alpha$ emission is attributed to star formation in the host galaxy, we can compute the SFR to be $S F R\left(M_{\odot} \mathrm{yr}^{-1}\right)=$ $7.9 \times 10^{-42} L_{\mathrm{H} \alpha}(\mathrm{erg} / \mathrm{s})($ Kennicutt et al. 1994). In our assumed cosmology, the measured $\mathrm{H} \alpha$ intensity transforms into a $\mathrm{H} \alpha$ luminosity $L_{\mathrm{H} \alpha}=(4.9 \pm 0.9) \times 10^{42} \mathrm{erg} \mathrm{s}^{-1}$. This implies a SFR (without any corrections) of $40 \pm 7 M_{\odot} \mathrm{yr}^{-1}$, which is much higher than the present-day rate in our Galaxy. From the total measured Ly $\alpha$ intensity (Castro-Tirado et al. 2002), we derive a Ly $\alpha$ luminosity $L_{\mathrm{Ly} \alpha} \leq(9 \pm 1) \times 10^{42} \mathrm{erg} \mathrm{s}^{-1}$, which implies a $S F R \leq 10 M_{\odot} \mathrm{yr}^{-1}$ in agreement with Fynbo et al. (2005) and Jakobsson et al. (2005). The $\mathrm{Ly} \alpha / \mathrm{H} \alpha$ ratio of 2 implies a low dust content in the GRB host galaxy, as also inferred by de Ugarte Postigo et al. (2005) and Kann et al. (2006) from the afterglow SED; there is also no excess absorption beyond the (low) Galactic value detected in Chandra X-ray observations (Sako \& Harrison 2002a). This is a high value for the SFR (see also Castro-Tirado et al. 2007), although the $S / N$ of the nIR spectrum implies a large uncertainty. The derived SFR implies that most of the ongoing star formation is unobscured, which is also in agreement with the SCUBA results at $850 \mu \mathrm{m}$ (Tanvir et al. 2004).

The Ly $\alpha$ photons produced in the ionized nebula experience a continuous resonant scattering in the presence of HI. In a dustfree nebula, this mechanism effectively traps the Ly $\alpha$ photons producing a typical P Cygni line profile in both emission and absorption, depending on the details of the density and velocity field. However, if even a little dust is present, most Ly $\alpha$ photons will disappear by heating dust grains, because radiation absorption by dust has a maximum cross-section close to the wavelength of Ly $\alpha$. Thus, the observed complex Ly $\alpha$ profile is the result of both mechanisms, resonance scattering which determines the profile shape, and dust absorption, which decreases yet further the intensity of the emission line. The Ly $\alpha$ P Cygni profile can be understood in terms of these mechanisms acting during the evolution of the swept-up HI supershell produced by a massive starburst in the host galaxy of GRB 021004. It corresponds to stage 4 in the evolutionary scheme developed by Tenorio-Tagle et al. (1999) and Más-Hesse et al. (2003), a stage compatible with GRB 021004 being the evolution endpoint of a WR star. 


\section{Conclusions}

We have identified several absorption line groups spanning a range of about $3000 \mathrm{~km} \mathrm{~s}^{-1}$ in velocity relative to the redshift of the host galaxy. The absorption profiles are very complex with both velocity-broadened components extending over several $100 \mathrm{~km} \mathrm{~s}^{-1}$ and narrow lines with velocity widths of only $\sim 20 \mathrm{~km} \mathrm{~s}^{-1}$. By analogy with QSO absorption line studies, the relative velocities, widths, and degrees of ionization of the lines ("line-locking", "ionization-velocity correlation") show that the progenitor had both an extremely strong radiation field and several distinct mass-loss phases (winds). These results are consistent with GRB progenitors being massive stars, such as LBVs or Wolf-Rayet stars, and provides additional insight into the nature of these progenitors and their immediate environments.

The host galaxy is a prolifically star-forming galaxy at a systemic redshift $z=2.3304$, which has a $S F R$ of $\sim 40 M_{\odot} \mathrm{yr}^{-1}$ as also found by Fynbo et al. (2005) and de Ugarte Postigo et al. (2005), reinforcing the potential association of some GRB with starburst galaxies (Christensen et al. 2004; Gorosabel et al. 2005, and references there in).

The Swift mission with a predicted lifetime of ten years (Gehrels et al. 2004) will certainly provide us the opportunity to carry out high-resolution spectroscopy for dozens of future GRBs, and to ascertain physical/chemical properties common to all GRB outflows.

Acknowledgements. We are grateful to M. Cerviño, M. Más-Hesse, R. González, G. Tenorio-Tagle and S. Vergani for fruitful discussions as well as the anonymous referee for the useful suggestions. This research has also been partially supported by the Spanish Ministry programmes AYA2004-01515, AYA 2007-06377, AYA 2009-14000-C03-01 and ESP 2002-04124-C03-01 (including FEDER funds). The Dark Cosmology Centre is funded by the DNRF. Some of the authors acknowledge benefits from collaboration within the EU FP5 Research Training Network "Gamma-Ray Bursts: An Enigma and a Tool".

\section{References}

Barraud, C., Olive, J.-F., Lestrade, J. P., et al. 2002, A\&A, 400, 1021 Berger, E., Frail, D. A., \& Kulkarni, S. R. 2002, GCN Circ., 1613, http://gcn.gsfc.nasa.gov/gcn/gcn3/1613.gcn3

Berger, E., Penprase, B. E., Cenko, S. B., et al. 2006, ApJ, 642, 979 Bersier, D., Stanek, K. Z., Winn, J. N., et al. 2003, ApJ, 584, L43 Björnsson, G., Gudmundsson, E. H., \& Johánesson, G. 2004, ApJ, 615, L77

Bloom, J. S., Perley, D. A., Li, W., et al. 2009, ApJ, 691, 723

Boroson, B., McCray, R., Oelfke Clark, C., et al. 1997, ApJ, 478, 638

Castor, J. I., Abbott, D. C., \& Klein, R. I. 1975, ApJ, 195, 157

Castro, S., Galama, T. J., Harrison, F. A., et al. 2003, ApJ, 586, 128

Castro-Tirado, A. J., Pérez, E., Gorosabel, J., et al. 2002, GCN Circ., 1635, http://gcn.gsfc.nasa.gov/gcn/gcn3/1635.gcn3

Castro-Tirado, A. J., Bremer, M., McBreen, S., et al. 2007, A\&A, 475, 101

Chen, H.-W., Prochaska, J. X., Ramirez-Ruiz, E., et al. 2007, ApJ, 663, 420

Chornock, R., \& Filippenko, A. 2002, GCN Circ., 1605, http://gcn.gsfc.nasa.gov/gcn/gcn3/1605.gcn3

Christensen, L., Hjorth, J., \& Gorosabel, J. 2004, A\&A, 425, 913

de Koter, A., Heap, S. R., \& Hubeny, I. 1997, A\&A, 477, 792

de Ugarte Postigo, A., Castro-Tirado, A. J., Gorosabel, J., et al. 2005, A\&A, 443, 841

de Ugarte Postigo, A., D’Elia, V., Goldoni, P., et al. 2010, A\&A, 513, A42

D'Elia, V., Fiore, F., Perna, R., et al. 2009, ApJ, 694, 332

Dessauges-Zavadsky, M., Chen, H.-W., Prochaska, J. X., Bloom, J. S., \& Barth, A. J. 2006, ApJ, 648, L89

D'Odorico, S., Dekker, H., Mazzoleni, R., et al. 2006, in SPIE Conf. Ser., 6269

Fiore, F., D'Elia, V., Lazzati, D., et al. 2005, ApJ, 624, 583

Fox, D. W., Yost, S., Kulkarni, S. R., et al. 2003, Nature, 422, 284

Fox, A. J., Ledoux, C., Vreeswijk, P. M., Smette, A., \& Jaunsen, A. O. 2008, A\&A, 491, 189

Frail, D. A., \& Berger, E, 2002, GCN Circ., 1574,

http://gcn.gsfc.nasa.gov/gcn/gcn3/1574.gcn3

Fynbo, J. P. U., Gorosabel, J., Smette, A., et al. 2005, ApJ, 633, 317

García-Segura, G., Mac Low, M.-M., \& Langer, N. 1996, A\&A, 305, 229

Gehrels, N., Chincarini, G., Giommi, P., et al. 2004, ApJ, 611, 1005
Gorosabel, J., Pérez-Ramírez, D., Sollerman, J., et al. 2005, A\&A, 444, 711

Gruendl, R. A., Chu, Y.-H., Dunne, B. C., \& Points, S. D. 2000, AJ, 120, 2670

Hjorth, J., Sollerman, P., Møller, P., et al. 2003, Nature, 423, 847

Holland, S. T., Weidinger, M., Fynbo, J. P. U., et al. 2003, AJ, 125, 2291

Humphreys, R. M., \& Davidson, K. 1994, PASP, 106, 1025

Jakobsson, P., Björnsson, G., Fynbo, J. P. U., et al. 2005, MNRAS, 362, 245

Jensen, B. L., Fynbo, J. P. U., Gorosabel, J., et al. 2001, A\&A, 370, 909

Kann, D. A., Klose, S., \& Zeh, A. 2006, ApJ, 641, 993

Kann, D. A., Masetti, N., \& Klose, S. 2007, AJ, 133, 1187

Kann, D. A., Klose, S., Zhang, B., et al. 2010, ApJ, submitted [arXiv: 0712.2186v2]

Kawabata, T., Matsumoto, K., Ayani, K., et al. 2004, IBVS, 5576

Kennicutt, R. C., Tamblyn, P., \& Congdon, C. E. 1994, ApJ, 435, 22

Klose, S., Greiner, J., Rau, A., et al. 2004, AJ, 128, 1942

Langer, N., Hamann, W.-R., Lennon, M., et al. 1994, A\&A, 290, 819

Langer, N., García-Segura, G., \& Mac Low, M.-M. 1999, ApJ, 520, L49

Lazzati, D., Rossi, E., Covino, S., Ghisellini, G., \& Malesani, D. 2002, A\&A, 396, L5

Lazzati, D., Perna, R., Flasher, J., Dwarkadas, V. V., \& Fiore, F. 2006, MNRAS, 372,1791

Maeder, A. 1983, A\&A,120, 113

Maeder, A., \& Meynet, G. 2000, A\&A, 361, 159

Más-Hesse, J. M., Kunth, D., Tenorio-Tagle, G., et al., Star Formation through Time, ed. E. Pérez, R. González-Delgado, \& G. Tenorio-Tagle, PASP Conf. Ser., 297, 177

Matheson, T., Garnavich, P. M., Foltz, C., et al. 2003, ApJ, 582, L5

Metzger, M. R., Djorgovski, S. G., Kulkarni, S. R., et al. 1997, Nature, 387, 878

Mirabal, N., Halpern, J. P., Chornock, R., et al. 2003, ApJ, 595, 935

Møller, P., Jakobsen P., \& Perryman, M. A. C. 1994, A\&A, 287, 719

Møller, P., Warren, S. J., Fall, S. M., Fynbo, J. P. U., \& Jakobsen, P. 2002a, ApJ, 574, 51

Møller, P., Fynbo, J. P. U., Hjorth, J., et al. 2002b, A\&A, 396, L21

Osterbrock, D. E. 1989, Astrophysics of Gaseous Nebulae and Active Galactic Nuclei (University Science Books, Mill Valley)

Pandey, S., Sahu, D. K., Resmi, L., et al. 2003, Bull. Astro. Soc. India, 31, 19

Pooley, G. 2002a, GCN Circ., 1575, http://gcn.gsfc.nasa.gov/gcn/ gcn $3 / 1575 . g c n 3$

Pooley, G. 2002b, GCN Circ., 1588, http://gcn.gsfc.nasa.gov/gcn/ gcn3/1588.gcn3

Prochaska, J. X., Chen, H.-W., \& Bloom, J. S. 2006, ApJ, 648, 95

Prochaska, J. X., Dessagues-Zavadsky, M., \& Ramirez-Ruiz, E. 2008, ApJ, 685, 344

Salamanca, I., Rol, E., Wijers, R. A. M. J., et al. 2002, GCN Circ., 1611, http://gcn.gsfc.nasa.gov/gcn/gcn3/1611.gcn3

Salvaterra, R., Della Valle, M., Campana, S., et al. 2009, Nature, 461, 1258

Sako, M., \& Harrison, F. A. 2002a, GCN Circ., 1624, http://gcn.gsfc. nasa.gov/gcn/gcn3/1624.gcn3

Sako, M., \& Harrison, F. A. 2002b, GCN Circ., 1716, http://gcn.gsfc. nasa.gov/gcn/gcn3/1716.gcn3

Sargent, W. L. W., Boksenberg, A., \& Steidel, C. C. 1988, ApJS, 68, 539

Savaglio, S., Fiore, F., Israel, G., et al. 2002, GCN Circ., 1633, http://gcn. gsfc.nasa.gov/gcn/gcn3/1533.gcn3

Schaefer, B. E., Gerardy, C. L., Höflich, P., et al. 2003, ApJ, 588, 387

Shin, M.-S., Berger, E., Penprase, B. E., et al. 2006, unpublished [arXiv: astro-ph/0608327]

Shirasaki, Y., Graziani, C., Matsuoka, M., et al. 2002, GCN Circ., 1565, http://gcn.gsfc.nasa.gov/gcn/gcn3/1565.gcn3

Smith, L. J., Pettini, M., Dyson, J. E., \& Hartquist, T. W. 1984, MNRAS, 211, 679

Srianand, R., Petitjean, P., Ledoux, C., \& Hazard, C. 2002, MNRAS, 336, 753

Stanek, K., Matheson, T., Garnavich, P. M., et al. 2003, ApJ, 591, L17

Starling, R. L. C., Wijers, R. A. M. J., Hughes, M. A., et al. 2005, MNRAS, 360, 305

Tanvir, N. R., Barnard, V. E., Blain, A. W., et al. 2004, MNRAS, 352, 1073

Tanvir, N. R., Fox, D. B., Levan, A. J., et al. 2009, Nature, 461, 1254

Tejos, N., Lopez, S., Prochaska, J. X., Chen, H.-W., \& Dessauges-Zavadsky, M. 2007, ApJ, 671, 622

Tejos, N., Lopez, S., Prochaska, J. X., et al. 2009, ApJ, 706, 1309

Tenorio-Tagle, G., Silich, S. A., Kunth, D., Terlevich, E., \& Terlevich, R. 1999, MNRAS, 309, 332

Thöne, C. C., Greiner, J., Savaglio, S., \& Jehin, E. 2007, ApJ, 671, 628

Uemura, M., Kato, T., Ishioka, R., \& Yamaoka. H. 2003, PASJ, 55, L31

Vergani, S., D., Petitjean, P., Ledoux, C., et al. 2009, A\&A, 503, 771

Vreeswijk, P. M., Ledoux, C., Smette, A., et al. 2007, A\&A, 468, 83

Wang, L., Baade, D., Höflich, P., \& Wheeler, J. C. 2003, unpublished [arXiv: astro-ph/0301266]

Woosley, S. E., \& Bloom, J. S. 2006, ARA\&A, 44, 507

Woosley, S. E. 1993, ApJ, 405, 273 